\title{
TTKC: A Taylor Triangle $K$ Coverage Algorithm in WSNs
}

\author{
Xiaohui Ji, Jie Xie and Yuanbo Li \\ School of Computer and Information Engineering, Luoyang Institute of Science \\ and Technology, Luoyang 471023, China \\ E-mail:78821389@qq.com
}

\begin{abstract}
Network coverage is a fundamental problem in wireless sensor networks; different scenarios for coverage of the network have different requirements, this article needle this issue presents a triangle geometric method for determining the characteristics of the target area coverage, and on this basis, designed $k$ degree coverage Algorithm (Taylor Triangle $k$-Coverage algorithm, TTKC). Firstly, the perception of each sensor node round draw into six equal triangular region, according to Theorem determine whether the region is to reach users on the network coverage requirements, then the algorithm scheduling the appropriate section Enter active to achieve the target of $k$-degree coverage area. Experimental results show that the TTKC algorithm to ensure network coverage quality conditions can be effectively drop Low number of active nodes to improve network energy efficiency, thereby extending the lifetime of the network.
\end{abstract}

Keywords: wireless sensor network; K coverage; network lifetime; Taylor triangle

\section{Introduction}

Wireless sensor network is a set of information collection, wireless communication in one The new network, can help users to remotely collect the information and the physical world multichip way to pass this information to the user, the physical world and the Information Society They will be linked together. Network coverage problem in wireless sensor networks A fundamental problem, it is mainly the reaction of sensor nodes deployed in how Effective monitoring of the target area, but also the network quality of service (QoS) One of the important evaluation index the pros and cons associated with the network coverage quality is the deployment of nodes. Unable to deploy section by artificial means in the harsh natural environment conditions when the point, the need to use tools such as aircraft deployed nodes, such the method known as random node deployment mode. Since this deployment the node position in advance uncertainty, this approach often need to deploy more Number of nodes, in order to protect the network robustness and fault tolerance, but it also brings node coverage control problem. At the same time, some important target area, as shown in Figure 1 on the military battlefield enemy tanks and other targets real-time monitoring, in order to achieve higher quality monitoring requirements, the need for the implementation of multi-target area coverage, this is the problem of multiple coverage, referred to covering issues $\mathrm{k}$ degrees. This paper mainly deals with stochastic network deployment k-degree coverage study the issue. Because of the manufacturing cost and size limitations node, the sensor node through often used to provide energy limited battery power source. In actual deployment when a node, due to the larger target area, the size of which requires network deployment larger, wider range distribution of nodes, the deployment environment is also more complex miscellaneous, leading through the replacement of the sensor node battery or charging other means to fill charging source is very difficult or very difficult to achieve. This problem can be transformed into the network lifetime problem, existing literature suggests solutions main there are two types: the first is density control node, which is mainly used 
in the network envelope initial planning stage; the second is the node status scheduling, this approach it is without degrading the performance of the system service conditions, by selecting a turn part of the node as an active node, and let the other nodes into a low-power sleep state, reduce the number of active nodes to improve network node energy utilization efficiency. This will be the node scheduling method designed based on k-degree coverage algorithm.

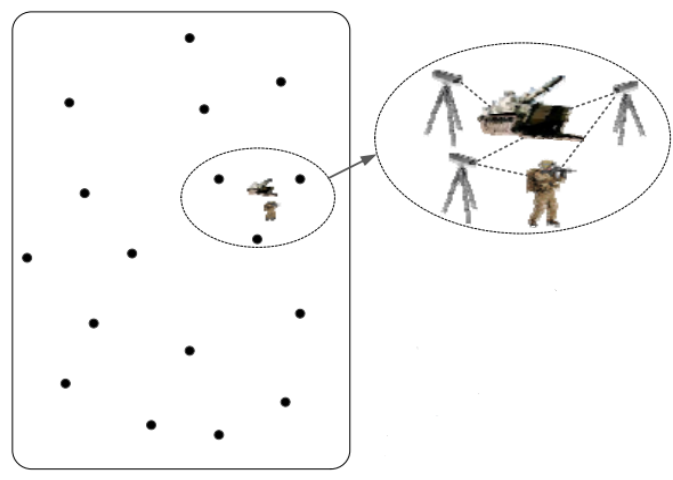

\section{Figure 1. Monitoring Application of WSN in Military Battlefield}

$K$ Coverage of a random node deployment under the coverage problem can be decomposed into two Sub-questions: First, in the range of network coverage, how to ensure that projects objectives of any one region at least in a sensor node within the scope of monitoring; second, when a user on the network coverage requirements increase, the how to schedule or mobile sensor nodes to achieve the target area more repeat cover. To solve the above problem, this paper presents a use Taylor triangle shaped geometric characteristics of the target area coverage of the identification method, then this based on the proposed algorithm based on $k$-degree coverage Taylor triangle (Taylor Triangle $k$-Coverage algorithm, TTKC), by scheduling the state of the corresponding node network coverage to reach quality requirements. The main contribution of this paper is:

(1) Analysis of the existing target coverage model, proposed uses Taylor three angular geometric characteristics of the target area coverage determination method and fixed management.

(2) Scheduling mechanism based on node status is designed $\mathrm{k}$ degree coverage count francs (TTKC), the algorithm has a low complexity, and good scalability.

(3) The simulation experiments, to the number of active nodes and network lifetime period of evaluation, by contrast CCP analyzed the proposed algorithm TTKC Performance.

Other paper is organized as follows. Section 1 describes the $k$-degree coverage the latest research progress issues; in Section 2 describes the network module type definitions and related issues; in section 3, is proposed based on Taylor $K$ of Los triangle covering algorithm; in Section 4, of the proposed operator law gives the experimental evaluation; and finally in Section 5 of this paper are summarized.

\section{Related Work}

$\mathrm{K}$ Coverage of the existing literature on the network nodes are randomly deployed covering problem research is mainly based on covering theory and node status scheduling strategy resolved. Solutions node cover set theory is in accordance with certain strategies part node, to meet completely cover the entire target area under conditions minimizing the size of the node set. Ammari et al. [1] In the node collection workers as "wheel" approach to study the problem of multiple coverage, through theoretical derivation was to achieve 
full coverage of the conditions of the network $\mathrm{k}$ proposed four configurations association proposed to solve the agreement able to elect the minimum number of nodes set and achieve $\mathrm{k}$ monitoring of coverage area; and in order to ensure network connectivity, literature while providing a node communication radius should remain proportional relationship with the induction radius department. On the basis of the above, based on the $\mathrm{k}$ of the network coverage issues forwarding node scheduling and data binding problem with the research, design a location-based data forwarding status agreement, the agreement is in accordance with the main according to different nodes sensing and communication range and select the appropriate size festival points, in order to achieve the degree of monitoring area coverage $\mathrm{k}$ [2]. Ashouri et al [3] it will be divided into a number of nodes deployed disjoint set of nodes, each node sets are able to cover the monitoring area alone, the use of Boolean satisfiability (SAT) theory to find the optimal set of nodes constructors, finally this extension method applications to solve the k-degree coverage. Luke moderate domestic scholars [4] proposed a species greedy method based on a minimum set cover approximation algorithm in structure covering set in the process, give priority to expand the largest active nodes join cover Lid cover set, the algorithm constructed a smaller scale, but the algorithm is not distributed, not suitable for energy constrained sensor networks. Solutions based on node status scheduling method are through a node position location information, select the appropriate node and schedule it to active state coverage of the target area. K-degree coverage of the study protocol is covered with classic set protocol CCP [6,7], which is the local position of nodes using the node information meets the coverage requirements determine eligibility functions, functions qualifiers will switch to the active state, without qualifiers will be converted to sleep. And based on covering theory Hall et al [5], presents a VORONOI figure geometric features to determine whether a node redundancy method, it first finds covering the circumference of the intersection between the nodes, as well as those covering the circumference of the network the intersection of network deployment border region; and then calculate the intersection of whether these bits in the circumferential extent of coverage node; if they have at least one point of intersection a cover disc cover, then the entire network deployment area was completely covered. If the intersection points are covered by at least $\mathrm{k}$ disc cover, that what area were deployed throughout the network node $\mathrm{k}$ degrees covered. However, section point function determines eligibility in determining whether a node redundancy algorithm complexity it reached the $\mathrm{O}(\mathrm{N} 3)$. Chinese scholars Wang strokes change [8] will ask k degree of regional coverage title into several specific points within the target area of coverage calculated $\mathrm{k}$ questions, put forward in order to ensure a degree of coverage for the target $\mathrm{k}$ centralized node scheduling policy, according to the time round, the algorithm determines a distributed fashion festival point state. Bejerano multiple for monitoring regional "coverage hole" coverage problems without relying on the local node position information condition, lift $\mathrm{K}$ cover a precise verification program, which can be achieved for different foot accurately detect inch hole cover [9].

\section{Problem Description and Analysis}

\subsection{Network Model}

Now assume that the network has the following characteristics:

(1) The sensor nodes deployed in a random form in the target area.

(2) Node after deployment can get its own location information section point position information by means of low-power GPS [11] or other location technologies [12] obtained.

(3) The node uses a disk perceptual model, namely the perception of the scope of the node it is its location in a circle, with a radius of Rs circular enclosed area, Rs called perception 
radius node, Rs by the physical size of the sensor characteristics Decisions. Deploy higher density

(4) Node which usually is the node most 2 to 3 times superior deployment situations.

\subsection{Basic Definition}

Definition1: the distance between any two nodes $d(i, j)$ are called nodes $i$ and $j$ Euclidean distance, when $d(i, j)<2 R$ referred to the neighbor node, node $\mathrm{i}$ and $\mathrm{j}$.

Definition2: in the monitoring of the target area, when a target node is $K$ sensor node coverage, called $K$ heavy cover.

Definition 3: in the monitoring of the target area, all sensor nodes coverage Union and all sensor nodes range and then, called network covering efficiency:

$$
E A=\cup S_{\mathrm{i}} / \Sigma S_{\mathrm{j}}
$$

Definition 4: Covering the region of coverage for:

$$
P\left(s_{\mathrm{i}}, s_{\mathrm{j}}\right)=e^{-\varepsilon d}
$$

Among them is sensor node physical parameters; Re said sensor node monitoring dynamic parameters in the said sensor nodes; $d\left(s_{\mathrm{i}}, s_{\mathrm{j}}\right)$ Euclidean distance; when $d\left(s_{\mathrm{i}}, s_{\mathrm{j}}\right)<\left(R_{s}-R_{e}\right)$, this time node $\mathrm{Si}$ is detected, it is not detected.

Definition 5: suppose that nodes $s_{i}, s_{j}$, the target regions they cover are $C_{i}$ and $C j$, and $C_{i} \cap C_{\mathrm{j}} \neq \varnothing$, so nodes $s_{i}$ and $s_{j}$ are coverage connected. Suppose that $T$ is a m nodes set randomly distributed in the target region, $\mathrm{E}$ is the set of edges of the network diagram, indicating that the $e_{\mathrm{ij}}=1$ positional relationship in $e_{\mathrm{ij}} ; e_{\mathrm{ij}}$ represents the positional relationship of node $s_{\mathrm{i}}$ and the target node tj. When $e_{\mathrm{i}}=1$ when and only when the Euclidean distance of target node $t_{\mathrm{j}}$ and the node si is less than or equal to the perception radius $r_{\mathrm{i}}$, otherwise $e_{\mathrm{i}}=0 . W=\left\{w_{1}, w_{2}, w_{3} \ldots w_{\mathrm{n}}\right\}$ is the initial energy set of sensor nodes; $W$ is in normal distribution; the $w_{\mathrm{i}}$ represents initial energy of sensor node $s_{\mathrm{i}} ; w_{\mathrm{i}}$ is the maximum energy in the process of node work.

Definition6: Taylor triangle: equilateral triangle with three vertices round heart, with its side radius for circle formed by the intersection of three circumferential region, called Taylor triangle. Gray area its shape as shown in Figure 2 domain.

According to definition, Taylor triangle is a fixed-width song line diagram to the basis of an equilateral triangle. Taylor arbitrary triangle point to distance its edges are not greater than the inscribed equilateral triangle sides long, each point corresponding to the distance to the edge of the vertices are equal.

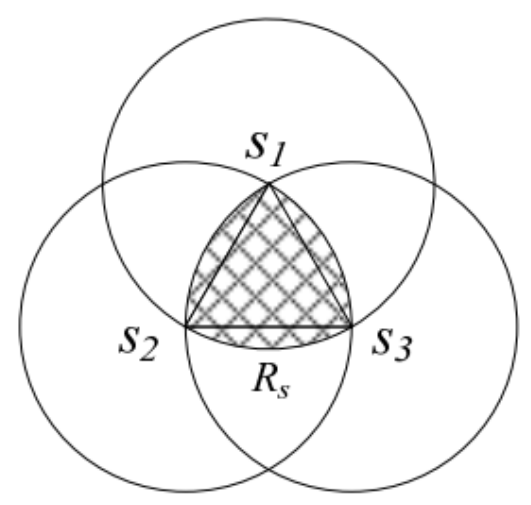

Figure 2. Taylor Triangle 


\subsection{Network Conditions and Theorem of K-coverage}

For more intuitively and effectively deploy the determination node of the target area the degree of coverage, use triangular geometric characteristics Taylor be covered determine the extent of the cover. The main idea is to achieve after the network initialization is complete, Helly theorems use [10] to determine whether the target is covered by a node $\mathrm{k}$ degrees.

Theorem 1: Assume that $\left\{X \mid X_{1}, X_{2}, X_{3} \ldots X_{n}\right\}$ is a finite subset of $R^{d}$, where $n>d$, if the sub-set of arbitrary $d n+1$ Intersection subset is not empty, then this collection has a non-empty intersection; namely $\cap X_{i} \neq \phi$.
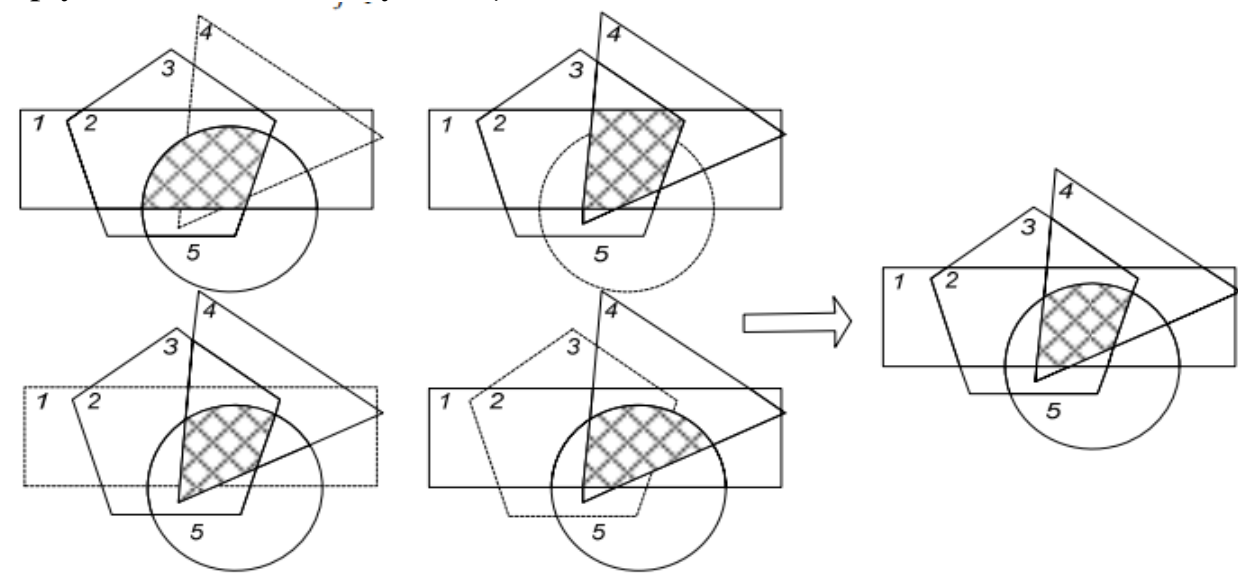

Figure 3. A Demonstration of Helly Theorem in 2-D surface

Proof: According to Theorem 1, in the $k \geqslant 3$ conditions, if and only if the $k$ geometry any three of the intersection is not empty, this geometry k formed by the intersection graph is a non-empty set. Figure 3 is a Helly set processing a sample on a two-dimensional plane, which represents the $n=5, d=3$ under pieces intersect each geometry formed by common intersection (area) love condition. Geometric properties and theorems from the Taylor triangle 1, can be obtained as analyzing the full article at the target area, or whether the target covered by the node $\mathrm{k}$ degree member.

Lemma 1: Under $\mathrm{k} \geq 3$ conditions, if in a triangle Taylor Shaped region in the $\mathrm{k}$-memory node is active, the Taylor three angular area covered by node $\mathrm{k}$ degrees.

Proof: According to the geometric definition of Taylor triangle, triangle in Taylor instance Taylor any form within the triangle sides point to its outside is not Taylor triangle is greater than the length of the side, while nodes have the same perception half diameter, if the Taylor triangle in the $k$ memory is active in node, then any node $\mathrm{k}$ in which one node to the triangle Taylor is not greater than the perceived distance between the edge of the radius of the node, that is, the Taylor triangle covered by the node $\mathrm{k}$ degrees. Thus, Lemma 1 conclusion holds proof. under conditions of actual deployment in a random form node to node unit department area is divided into several adjacent triangles Taylor coverage of goals judging is more difficult, therefore this paper the following solutions, first, divide the circumference of a sensor node into six equal sectors Field, as shown in Figure 4 (a). Each sector contains a fan by the connection with the two sides equilateral triangle formed by the intersection of the arc and the chord formed by two edges shape, in each equilateral triangle constructed corresponding Taylor triangle, so that any two adjacent triangular-shaped intersection Taylor form one pair arc zone, as shown in 4 (b) below. Then double arc area for this article domain judgment node coverage area. 


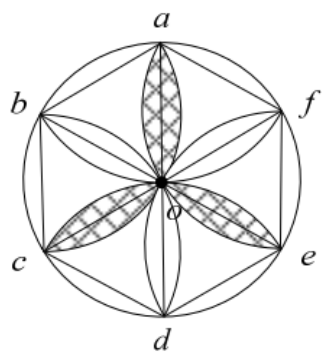

(a)

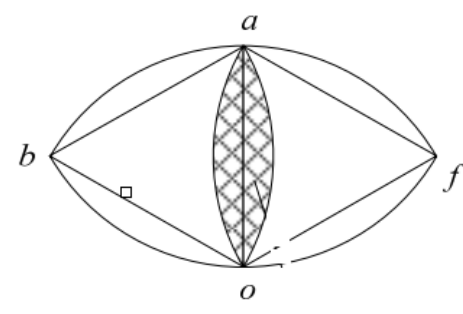

(b)

Figure 4.2. Arch Area Formed by Two Adjacent Taylor Triangle

Lemma 2: Under $k \geq 3$ conditions, if with the same common base double curved edge region two Taylor triangle formed by memory in the $\mathrm{k}$ active nodes, then the two adjacent triangular areas are nodes Taylor k-degree coverage.

Proof: The double curved region is that the two triangles of public Taylor Area, known by the Lemma 1, double curved arbitrary point distance between these two Taylor any triangle edge that is not greater than the length of the side and the two Taylor triangle is equal, and therefore, if the pair of arcuate area of memory in the $\mathrm{k}$ active nodes, then the two triangles can be that $k$ Taylor node coverage QED.

Theorem 2: Under the conditions $k \geq 3$, for a target area, such as if at any Taylor triangle within a triangle adjacent thereto Taylor double arc-shaped area formed by the memory in the $k$ active node and Taylor perception radius triangle straight edge length equal to the node size, then this an area covered by node $k$ degrees.

Proof: assuming that the region is divided into a number of equal and adjacent the equilateral triangle, sensing the size of its radius equal to the side length of the node, if from these equilateral triangle constructed Taylor triangle and its neighboring the double curved area Taylor triangle formed in the $k$ active memory section point by Lemma 2 know, these two triangles is node Taylor Area $k$-degree coverage. Similarly, if there are other double curved region $k$ live Jump node, this dual arc where the two triangles are also sections Taylor Point $k$-degree coverage, thus concluding the establishment. QED. In order to determine the connectivity between nodes, Theorem 3 below, it reflects the condition of ensuring network coverage, between network nodes to achieve interconnecting node communication radius and the induction radius should satisfy constraints condition.

\section{TTKC Algorithm and Evaluation}

Due to the connectivity of high density sensor nodes and the coverage area of target nodes, the randomly-given coverage area is reconstructed through limiting the maximum amount of distortion of randomly covered area in a certain range in order to maximize life cycle of the network and solve the optimization problem better. At this point, the maximization of network lifetime is transformed into the problem of target area coverage. In essence, the maximization of given network lifetime is transformed into seeking the largest set of sensor nodes in the entire target area. Each sensor node is related to the specific coverage area required by nodes in target region. Therefore, the optimization can be solved. After long-time study, many experts and scholars found that there're two main factors affecting the network lifetime: First, the energy consumption of each sensor node in the process of collecting data. Second, the topological geometry of deploying had sensor nodes. Due to the transformation from the optimization of network lifetime into the coverage problem, it is particularly important for sensor nodes to cover specific areas, which is the key to solve the optimization problem. In this paper, we first propose to 
transform the information collection and data retrieval into energy restriction and coverage-related issues. Second, the given network lifetime maximization problem is determined as a NP-complete problem. Third, the scheduling mechanism of sensor nodes is transformed while the optimal coverage rate is solved through the greedy algorithm. The energy consumption is effectively decreased and the network lifetime is prolonged. The energy consumption of sensor nodes had in data retrieval. In the whole network system is solved finally, the effectiveness and applicability of the algorithm is verified through experimental comparison.

Step 1: Set the position and coverage radius $r$ of sensor nodes. The entire coverage region is divided into multiple disjoint sub-regions $\left\{F_{1}, F_{2,}, F_{n}\right\}$. Sensor nodes in every sub-region $F_{i}$ and all coverage regions are connected and guaranteed in the region, i.e. $F_{l}=\left\{S_{n 1}, S_{n 2} \cdots S_{n l}\right\}, \quad F_{l} \subset M_{s i}$. Every related subset is marked as $S_{i}=\left\{F_{n 1}, F_{n 2} \cdots F_{n l}\right\}$ and $\bigcup_{i=1,2 \cdots n} F_{i}=A$.

Step 2: As for the $i(\mathrm{i}=1,2 \ldots \mathrm{n})$ data retrieval, its output is a coverage set: $C_{i}$. A concerned sub-region $F_{c}=\underset{F_{i}}{\arg \min } \sum_{S_{n i} \in E_{i}}\left\lfloor E_{r}^{i} / E_{c}^{i}\right\rfloor$ is selected. $E_{r}^{i}$ is the remaining energy of the $i$ sensor node in the current data collection and retrieval.

Step 3: In order to reduce the amount of overlapping coverage for sparse coverage sub-region caused by the redundant nodes, so an overlap value $v_{i}=F_{c}$ for the $i_{\text {th }}$ sensor node is defined. It represents the number of sensor nodes and $F_{c} \in S_{i} . v_{i}$ is the number of selected important sub-areas for the coverage of the $i_{\mathrm{th}}$ sensor nodes, i.e. $v_{i}=v_{\min } \cdot v_{\min }$ is the minimum number of sensor nodes in the covered target area.

Step4: All the sensor nodes in the current coverage area update the remaining energy by reducing the consumed energy in the previous data collection and retrieval from the remaining energy, written as $E_{r}^{i}=E_{r}^{i}-E_{c}^{i}$. If the remaining energy of a sensor node is less than the required energy for one data collection and retrieval, the sensor node should be removed from the sensor set. Then go to Step 2 and continue until coverage region $A$ is not fully covered by sensor nodes available any longer. At this time, the network lifetime is maximized.

\subsection{Determination of the Node Probability}

In the designated monitoring area, when any node receives data packets transmitted by the rest $N-1$ nodes, it can be considered to finish data retrieval.

Theorem 3:In the wireless sensor network constructed by $N$ wireless sensor nodes of one monitoring area, when there is one and only one sensor node transmitting the data packet and the probability value of the node $p_{i}$ is $1 / N+1-i$, the value of $p_{i_{-} \max }$ is the maximum one.

Proof: In the monitoring area, let all the sensor nodes are independent, there is one and only one sensor node working probability marked as $\lambda$. At the time $t_{0}$, moment, when all the sensor nodes $N$ work simultaneously, coverage probability of one sensor node is:

$$
p_{i}=C_{N}^{1} \lambda(1-\lambda)^{N-1}
$$

The probability event complies with geometric distribution of the required time, so in this monitoring area, when $N+1-i$ nodes are working, the successful coverage probability of a single node for monitoring the area is:

$$
p_{i}=(1-\lambda) C_{N-i}^{1} \lambda(1-\lambda)^{N+1-i}=C_{N-i}^{1} \lambda(1-\lambda)^{N-i}
$$

According to the definition of geometric distribution, the probability event in equation (4) still complies with the geometric distribution of the required time.

Taking the derivative of $\lambda$ on the left of formula (4) and make its result is 0 , we get:

$$
C_{N-i}^{1}(1-\lambda)^{N-1-i}[1-\lambda-(N-i) \lambda]=0
$$

Discussion: In the first case, according to the probability theory, the probability value is 
non-negative and not larger than 1, i.e. $\lambda \in[0,1]$. when $\lambda=1$, the single sensor node can the cover the monitoring area completely, which is contradictory with $N+1-i$ nodes involved in working, so $\lambda \neq 1$; In the second case, when $\lambda=0$, the single sensor node is in the sleeping or dead state, which is also in contradiction with the meaning, so $\lambda \in(0,1)$.

Let $1-\lambda-(N-i) \lambda=0$, so $\lambda=1 / N+1-i$. When $\lambda=1 / N+1-i, p_{i}$ gets the maximum value. Let: $u=N-i+1$, i.e., $\lambda=1 / u$ and get it in the formula (5). The following can be obtained:

$$
\begin{aligned}
p_{i} & =\frac{(u-1)\left(1-\frac{1}{u}\right)^{u-1}}{u}=\frac{(u-1)(u-1)^{u-1}}{u^{u}}=\left(\frac{u-1}{u}\right)^{u} \\
& =\left(\frac{N-i}{N+1-i}\right)^{N+1-i}
\end{aligned}
$$

\subsection{Evaluation of Performance}

In order to study this subject better, the meaning of each parameter is listed one by one:

$1:$ the side length of a square

$\Omega:$ the area for a square, i.e. $\Omega=l^{2}$

$n:$ the number of randomly deployed sensor nodes

$r$ : the sensing radius of sensor nodes

$E(C)$ : the expectation of coverage area of sensor nodes, i.e. $\mu$

$\sigma^{2}$ : the variance of coverage area of sensor nodes

$p(x)$ : the coverage rate of randomly deployed sensor nodes

In order to improve the evaluation of network performance, MATLAB6.5 is used in simulation experiment. Coverage and connectivity of network in different scales can be realized through changing range of the coverage region. The model performance in different scales can be evaluated better. It is mainly reflected in the minimum number of nodes deployed in the cases of different coverage and network connectivity rate. The average value is derived from simulations of 100 times. The curve for node coverage variation in different network scales is shown in Figure 5:

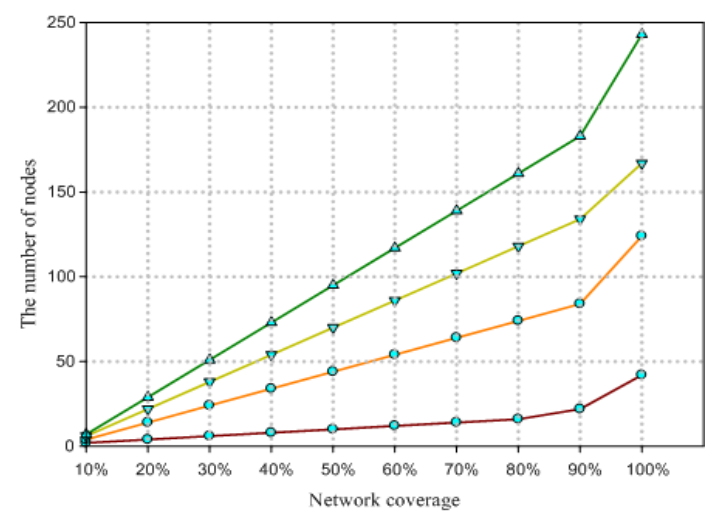

Figure 5. Curve for Node Coverage Variation in Different Network Scales 
As can be seen in the Figure 5, firstly, there are fewer nodes for complete coverage in the network with smaller area and more nodes in larger area. For example, when coverage is $100 \%, 43$ nodes are needed in $100 * 100$ area; 124 nodes in $200 * 200$ area; 167 nodes in $300 * 300$ area ; 243 nodes in $400 * 400$ area. Secondly, the number of sensor nodes is not the same according to different requirements of networks for coverage rate. The number of nodes is increasing as the network grows, which shows a linear increasing relationship. In Figure 5, it requires more nodes to realize full coverage for much larger networks and fewer nodes for smaller networks. When the coverage rate is between $90 \%$ and $100 \%$, the increment rate of large network had increases faster than that of small-scale networks.

To further verify the coverage rate of WSN in probability model, the $100 * 100$ model is chosen for study. After the network parameters are given dynamically, the proportion between network coverage rate and the number of nodes is compared, as is shown in Figure 6:

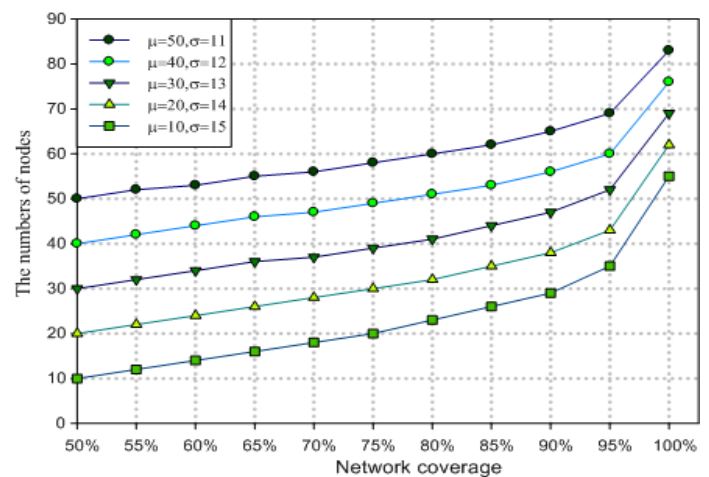

Figure 6. Curve for Network Coverage Variation with Different Parameters

Figure 6 shows the network coverage rate under different parameters, its computation process is according to formula (20), when stochastic variable follows normal distribution $N \in\left(\mu, \sigma^{2}\right), \quad p(X \leq b)=\phi((b-\mu) / \sigma)$ is used to get the result. Take $\mu=10, \sigma=15$ as an example, when the coverage rate is $60 \%, p(X \leq b)=\phi((b-10) / 15) \geq 60 \%$, it can be deducted that $((b-10) / 15)=0.255$, and $b=\lceil 13.825\rceil=14 . \mu=10, \sigma=15$, when the coverage rate is $60 \%$, the required number of nodes is 14 . From Figure 6, we can also see that, in case of same network size, the larger the expectation, the more nodes will be required, i.e. The normal distribution model that the network shows. For any curve, the variation is linear relationships that is because, the first: the coverage rate increase at 5\%, so the distributed function values changes slowly. Second, the difference of expectation in the example is 10 , which means the two adjacent curves have the feature of equal spacing. Third, when the coverage rate exceeds $95 \%$, the number of nodes increased significantly. That is due to the quick change of distributed function values between $95 \%$ and $99.9 \%$. For example, if the expectation is 10 and variance is 15,35 nodes are needed with coverage rate $95 \%$ while 55 is needed with coverage rate $99.9 \%$.

For another important factor of WSN connectivity, the size of connectivity is directly related to the performance of data transmission, data processing and data computation, etc. Next four different network models are adopted to compare the connectivity of WSN in experiments. According to formula (3), (4), and (5), the number of sensor nodes is in different network models are solved, as is shown in Figure 7: 


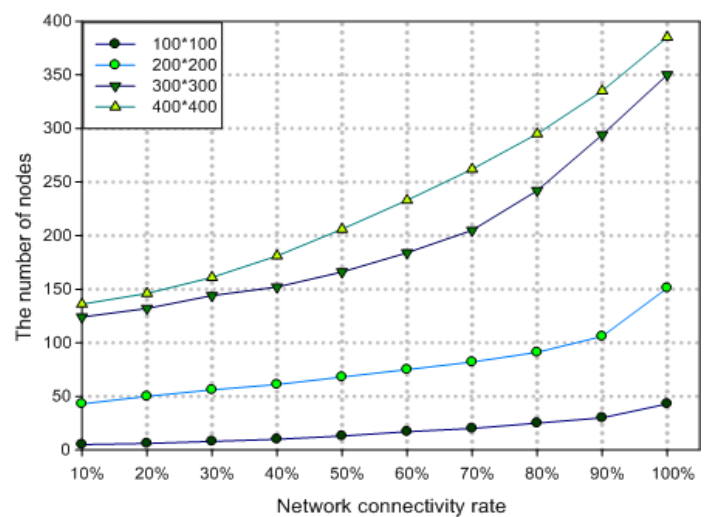

\section{Figure 7 Curve for Network Connectivity in Different Network Models}

Figure 7 reflects the variation curves between network connectivity rate and the number of nodes. For the two networks of $100 * 100$ and $200 * 200$, the number of nodes increases more slowly. The main reasons are: First, when the network is small and the connectivity rate is $100 \%, 48$ nodes can complete the connectivity between nodes in the network model of $100 * 100$ while 150 nodes are needed in the network model of $200 * 200$. Second, for the two network models, the increasing trends of the nodes number are relatively stable. The node number increments are in linear relationship with time. For the two networks of $300 * 300$ and $400 * 400$, because their areas are larger, more nodes are needed compared with the previous two network models. At the beginning, the nodes number in the two network models are almost the same. However, with the expansion of the network model, the nodes are greatly increasing in $400 * 400$ network model, because in the communication process, the optimal communication rule is the two circles of sensor nodes are circumscribed, which is, however, impossible to achieve. Therefore, as the network grows, much more nodes are largely required.

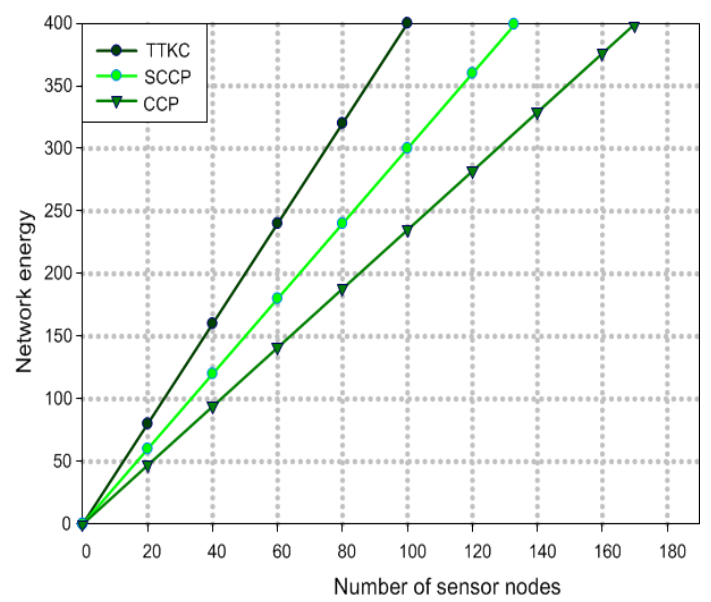

Figure 8. The Relationship between Number of Sensor Nodes and Network Energy 


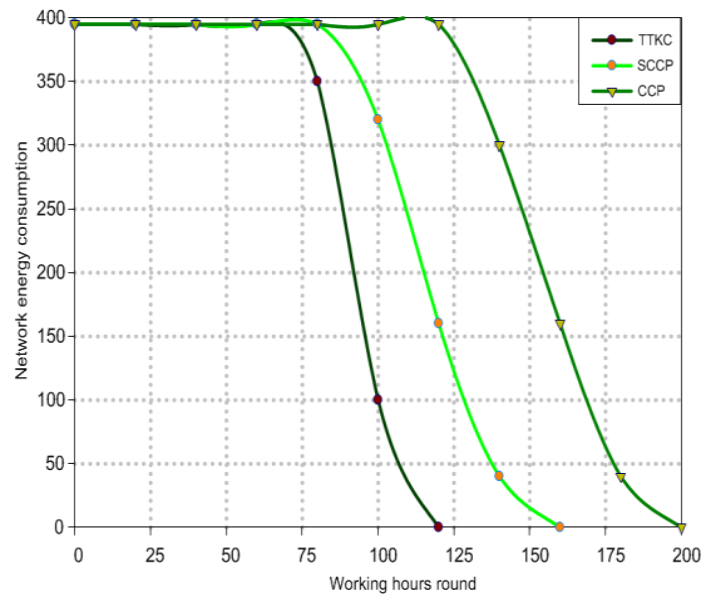

Figure 9. Curve for Node Energy and Time Rounds

Figure 8 and Figure 9 shows the comparison between network energy and nodes number along with the rest energy of nodes changing with time for the TTCK algorithm and algorithms of CCP and SCCP with the precondition of network coverage. As can be seen from the figure, with the same nodes number, the progressively increased speed of TTCK algorithm is larger than that of the algorithms of CCP and SCCP. For a certain energy value, the nodes of TTCK algorithm are much less than that of the other two algorithms. Figure 9 reflects the total remaining energy of network nodes is decreased gradually with time in the operation of system. Compared with the other two algorithms, less energy is consumed while the network coverage can be ensured through this algorithm. After the network runs for the same period of time, $9 \%$ energy in average can be saved by using this algorithm than SCCP and 17\% energy than CCP. This is because it costs little in the calculation of network coverage and less energy of sensor nodes is consumed.

\section{Conclusion}

This paper researches the coverage algorithm in wireless sensor networks and presents TTCK algorithm with the theory of probability. First, TTCK establishes the relation model and association relation between sensor nodes and target nodes. Then, the calculation of a target node's trajectory is given based on its probability and expectation. Next, target nodes can be covered more effectively through scheduling mechanism of nodes. The simulation results show TTCK is effective and scalable. In future research, TTCK will be extended to implement the multiple coverage are in heterogeneous wireless sensor network. Furthermore, we will study how to do quadratic linear programming for nodes with random distribution and improve the calculating precision for the border coverage.

\section{References}

[1] C. Schuragers, V. Tsiatsis, S. Ganeriwal and M. Srivastava, "Optimizing sensor networks in the energy-latency-density design space”, IEEE Trans. Mobile Computer, vol. 1, no. 70, (2002).

[2] H. Thomas, S. Yi, D. Hanif and D. Sherali, "Rate allocation and network lifetime problems for WSN", IEEE/ACM Trans. Networking, vol. 16, no. 321, (2008).

[3] H. Shibo, C. Jiming and S. Youxian, "Coverage and connectivity in duty-cycled WSNs for event monitoring”, IEEE Trans. Parallel and Distributed Systems, vol. 23, no. 475, (2012).

[4] S. Zeyu, W. Weiguo and W. A. Huanzhao, "Novel Coverage Algorithm Based on Event-Probability-Driven Mechanism in Wireless Sensor Network", Journal on Wireless Communications and Networking, vol. 13, no. 1, (2014).

[5] W. Li and W. Zhang, "Coverage analysis and active scheme of WSNs", The Institution of Engineering and Technology, vol. 2, no. 86, (2012). 
[6] L. Kai, J. Joel, M. Rodrigues, G. Hongwei, X. Naixue and L. Xuedong, "Energy efficiency QoS assurance routing in WSN", IEEE Systems Journal, vol. 5, no. 495, (2011).

[7] T. M. Chiwewe and G. P. Hancke, "A distributed topology control technique for low interference and energy efficiency in WSN", IEEE Trans. Industrial Informatics, vol. 8, no. 11, (2012).

[8] T. C. Sheng, S. S. Jia, and Y. C. Tun, "MLPA-conservation mechanism in WSN environments", EURASIP Journal on Wireless Communications and Networking, vol. 16, no. 1, (2012).

[9] R. Hamid, Y. Karkvandi, R. Efraim and Y. Orly, "Effective Lifetime-Aware routing in WSNs", IEEE Sensors Journal, vol. 11, no. 3359, (2011).

[10] S. Zeyu, W. Weiguo, W. Huanzhao, C. Heng and W. Wei, "An Optimization Strategy Coverage Control Algorithm for WSN", International Journal of Distributed Sensor Networks, vol. 23, no. 1, (2014).

[11] X. Tang and J. Xu, "Optimizing lifetime for continuous data aggregation with precision guarantees in WSN", IEEE/ACM Trans. Networking, vol. 16, no. 904, (2008).

[12] H. C. Fan and L. Mingyan, "Randomly Duty-cycled WSNs: dynamics of coverage", IEEE Trans. Wireless Communications, vol. 5 , no. 3182, (2006).

[13] W. Bang, S. K. C. Vikram and W. Wei, "Information coverage in randomly deployed WSN", IEEE Trans. Wireless Communications, vol. 6, no. 2994, (2007).

[14] X. Yang, W. H. C. Kui, S. Bo, Z. Ying, S. Xinyu and L. Chong, "Coverage and detection of a randomized scheduling algorithm in WSNs", IEEE Trans. Computers, vol. 59, no. 507, (2010),

[15] S. Zeyu, W. Huanzhao, W. Weiguo and X. Xingfei, "ECAPM: An Enhanced Coverage Algorithm in Wireless Sensor Network Based on Probability Model", International Journal of Distributed Sensor Networks, vol. 24, no. 1, (2015).

[16] D. Tian and N. D. Georganas, "A Node scheduling scheme for energy conservation in large WSNs", Wireless Communications and Mobile Computing, vol. 3, no. 271, (2013).

\section{Authors}

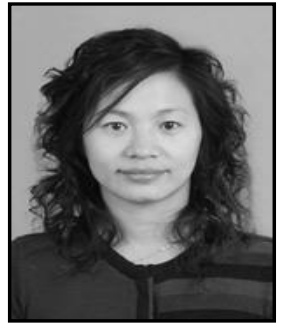

Ji Xiaohui, she was born in Luoyang City, Henan Province, a lecturer of Luoyang Institute of Science and Technology Computer and Information Engineering Department. Her main research interests include artificial intelligence and parallel.

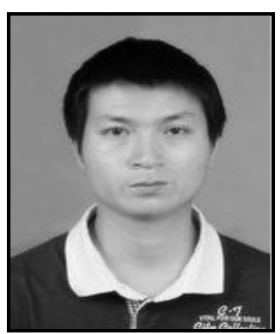

Xie Jie, he born in Luoyang City, Henan Province, a lecturer of Luoyang Institute of educational administration department. His main research interests include semantic Web, Web service, and data mining. 